\title{
The actual questions of the realization of the personality-oriented educational paradigm in the context of digitalization
}

\author{
Irina Kameneva* \\ South Federal University, 344006, Rostov-on-Don, Russia
}

\begin{abstract}
In the context of digitalization, the existing educational paradigms are being transformed. Transformational processes are associated with the solution of essential problems at key nodes that are significant for the construction of the entire paradigmatic series. As such, the nodes in this study, the values, goals, and results of education, the content of education were considered, and the issues of implementation of the student-centered educational paradigm that was relevant for them in the context of digitalization were identified. The most important are the questions about the image of a person, which determines the direction of pedagogical efforts, about the place of eternal universal values rooted in human culture, among other value orientations of education, about the criteria for the selection of content and the representation in its structure of the personal experience of students and teachers, about didactically significant specifics of electronic media of content. The dialogue character of the educational content and interaction in the educational process, determined by the culture, is emphasized. A comparative analysis of knowledge-oriented, personality-oriented, and digital educational paradigms in the given key nodes has been carried out. The role of the teacher as a subject of a personality-oriented educational process, acting here in two guises: professional and personal, is noted. The problems and risks associated with the weakening of the personal approach in education are formulated.
\end{abstract}

\section{Introduction}

Research of the current educational practice exposes the problem of transformation of educational paradigms in the context of digitalization. Especially sensitive to the factor of digitalization of education becomes person-oriented paradigm: the cooperation of the subjects of the educational process that realize through the digital media reduces the personality aspect. There comes a danger of technocratization of both learning and teaching activities, and the result is the losing of subjectivity by the participants in the educational process. However, while maintaining the anthropocentricity of the educational vector in the context of digitalization, a personality-oriented approach can effectively realize its

* Corresponding author: iykameneva@sfedu.ru 
teaching, developmental and educational potential, using the multimedia of the digital environment, interactivity, modeling capabilities in the digital space, etc.

Digitalization of education can be described as a transformational transition, caused by the 4th industrial revolution and determining the changes of all life spheres that pertinent to it, including the educational sphere. This largely explains the focusing of research on the role and significance of digital technologies and media.

The ambivalence of the assessment of their integration is clearly expressed. On the one hand, they are becoming new "culture tools" [1], it comes necessary to make a new educational ecosystem [2], and digital education is touted as s new educational paradigm. The structure of the teacher's professional competence, digital competencies, and the extent to which he initiates digital learning activities are especially factored [4]. On the other hand, digitalization is regarded as an attack on human culture. There are too many educational risks causing dehumanization of people and substitution of real values with imaginary ones $[5,6,7]$.

At this stage of the discussion, it seems a priority to research the problematics of digital education from a didactic perspective $[8,9]$. Especially important to consider the scientific views about the development of personality in the educational process [10].

\section{Materials and Methods}

The main goal of our research is to elucidate the actual questions of the realization of the personality-oriented educational paradigm for the keynote aspects such as values, goals, results of education, and content of the educational process, in the context of the transformational process of digitalization. The hypothesis of the study was the assumption that the establishment of didactically significant issues of the implementation of a personality-oriented educational paradigm, relevant in the context of digitalization, allows one to assess the possibilities, measure, and risks of using digital technologies in the educational process focused on the development of a person's personality.

This research uses the axiological approach and principles of the paradigm approach. The methods of generalization, systemic and comparative analysis, synthesis, and interpretation are used. The author analyzes the personality-oriented, knowledge-oriented, and digital educational paradigms. The methodological basis of the research is the concept of personality-oriented education, the cultural and historical concept of the development of the psyche and personality development, the dialogic concept of culture, the socio-cultural concept of the content of education. The sources of the study were two groups of scientific works: publications devoted to conceptual issues of knowledge-oriented, student-oriented, and digital education $[1-3,5,6,11,12]$, and publications considering the specifics of the use of digital technologies in the educational process $[4,7-10,13,14,15]$. The research was carried out in two stages. At the first stage, the sources were analyzed, and it was indicated that such key nodes as "values", "goals", "results" of education, "content" of the educational process are decisive concerning such nodes as "technology", "methods", " forms "of the educational process. The didactically significant issues of the implementation of the personality-oriented paradigm are highlighted. At the second stage, didactically significant issues of the implementation of the personality-oriented paradigm, relevant in the conditions of digitalization of education, were established, and the degree of their scientific development to date was revealed.

\section{Results}

\subsection{Values, goals, and results of education}


When getting acquainted with the digital concept of education, attention is drawn to the emphasis on the consideration of the rigid connections between the didactic categories "goal" - "results". However, goals didactically mediate values that must be reflected in the result. Conceptual is the correlation of "values" - "results". Therefore, it is necessary to consider this key node using the three-term formula: "value - goal - results".

The personality-oriented (or personality-developing) educational paradigm rightfully has the status of humanistic, since the highest value here becomes the person himself, with his unique personality and life history, with his unique personal experience acquired in situations of free personal choice. The goal of personality-oriented education is the development of a person as a free, humane personality, acquiring its spiritual essence by comprehending the culture and creating it (E.V. Bondarevskaya), joining the highest human values. With this type of education, not only the demand of society, the state for education, but also the demand of the individual himself is considered. The upbringing process is "sewn" into the learning process.

The personality-oriented paradigm is an attempt to resolve contradictions and eliminate the limitations of the knowledge-oriented educational paradigm, which represents in scientific theory a phenomenon called traditional learning. The value, in this case, is not the person himself, the totality of knowledge, skills, and abilities mastered by him, the performance of assigned functions, behavioral models, etc. Accordingly, the goal of knowledge-based education is the formation of a certain type of personality (depending on the requirements of social request). The educational process occurs in the unity of teaching and upbringing, which sets benchmark values and norms.

The knowledge paradigm is definitely an older paradigm of education. However, the ideas of the personal orientation of education and upbringing have been known to humanity since Antiquity (sufficing to recall the pedagogical ideas of Socrates that in person it is necessary to awaken the desire for self-knowledge, for the search for his moral truth, as well as a dialogue method, the use of which is organic for a personal approach to education since a person lives and develops only in dialogue).

Digital education, which claims the status of a new educational paradigm today, considers a person as a bearer of competencies with a set of hard (subject, professional) and soft skills. The core of the soft skills is made up of critical thinking, creativity, communication, and collaboration, opposed to the skills of reading, writing, and counting, which in this comparison are called the basic skills of traditional education [11]. The individual educational trajectory built as a result of the analysis of the data of the introductory diagnostics (psychotype, motivation, level of competence formation, etc.) should create conditions for the development of abilities leading to a particular student and in demand in the current labor market.

\subsection{The content of the educational process}

The content of education is that material and spiritual substance which the learner acquires in the educational process. There are three questions within the theme of this article, waiting for their principled solving to realize the personality-oriented paradigm in the context of digitalization:

- the question of the criteria for the selection of content,

- the question of the place of personal experience in the content,

- the question of the way of presentation and types of content carriers.

In the knowledge-oriented educational paradigm, the content is the system of knowledge, acquirements, and skills tested towards the studied subject area, didactic and psychological science, meeting the criteria of compliance with the modern level of 
development of science, society and production, age characteristics of students, proportionality, and unity of all structural units of educational content, etc.

Personal experience is either not included at all in the content of the educational process, or is included optional, as an illustration of what is being studied. The content is a monologue (the presentation of the material occurs from one point of view, or several points of view are presented, but one is initially designated as the only correct one), is presented mainly in educational books (textbooks, teaching aids) and the teacher's speech, and unfolds as a linear text. An educational book can exist on paper and electronic media. The teacher's speech within the framework of the knowledge approach can also be transferred to the space of electronic interaction.

In the personality-oriented paradigm, knowledge, which does not lose its fundamental significance and is selected following well-known scientific and didactic criteria, ceases to be a student's goal and purpose and becomes a means of personality development. Since the personality develops only in an activity (A. N. Leont'ev) and this activity is always fundamentally dialogical, the content of the educational process dialogizes and consists of at least three components, two of which include content that is external to the student (different points of view, scientific theories, etc., potentially able to enter a dialogue), and the third is his personal experience. The points of view that fill the dialogue components do not exist on their own but in the context of different cultures (cultures of different eras, peoples, cultures of thinking, specific of different subject areas: mathematical, physical, etc., and rest). The educational book becomes auxiliary, a book's weight of original cultural texts is increasing in the structure of the content (fiction, natural science, texts of original documents, etc.). The student deals with linear texts. Identical to educational books, cultural works can be presented on electronic media. The teacher's speech is also dialogized. The lecture is often criticized for its monologue. However, if it is didactically transformed into a dialogue, then it will use the students' inner speech and inner dialogue. This lecture often has an interactive component (for example, questions directed to the audience). Besides, the teacher's personal experience is integrated into the content of the educational process in the context of a personal approach. The teacher plays two roles here (which are integrated into his subjectivity): professional, as a designer of the educational process (starting with the organization of the content) and personal, as one of the participants in the dialogue, bringing to it his meaning, determined by his personal values. In fact, we can talk about a minimum four-component structure of the content, where the fourth component is the teacher's personal experience.

The digital educational paradigm assumes the greatest variability of educational content compared to the two previous paradigms. It is obvious, that the content field enlarges during the digital transformation, which is done by addressing the list of separate and batch educational materials, presented on different digital educational platforms, e.g. universities' databases, educational resources in social networks, etc.

The selection of content should be carried out depending on the characteristics of the student's individual profile. However, engaging personal experience in this field is not guaranteed. Content can be presented both linearly and non-linearly. All engaged texts are usually located on digital media. These can be hypertext, multimodal and regular linear texts. The teacher accompanies the student through his individual educational trajectory, initiating digital learning activities. There are three levels at which higher education teachers are currently initiating digital learning activities (it seems to us that this data can also be extrapolated to middle-level teachers): low, moderate, and high. And accordingly, teachers are divided into three clusters. Table 1 below by researchers Lohr, A., Stadler, M., Schultz-Pernice, F., Chernikova, O., Sailer, M., Fischer, F., Sailer, M. shows the number of higher education teachers as well as the mean scores of initiated digital learning activities in each of the three clusters. Higher education teachers in Cluster 3 (high level) initiated 
digital learning activities at a high level, as they initiated all four digital learning activities with a similar frequency [4].

Table 1. Descriptive results (N, Mean) from the cluster analysis for the four types of initiated digital learning activities: passive, active, constructive, and interactive [4].

\begin{tabular}{|c|c|c|c|}
\hline & $\begin{array}{c}\text { Low level } \\
\text { (Powerpointers) }\end{array}$ & $\begin{array}{c}\text { Moderate Level } \\
\text { (Clickerers) }\end{array}$ & $\begin{array}{c}\text { High Level } \\
\text { (Digital Pros) }\end{array}$ \\
\hline $\mathrm{N}$ & 483 & 477 & 437 \\
\hline Passive (M) & 1.54 & 3.39 & 2.48 \\
\hline Active (M) & .92 & 2.69 & 2.60 \\
\hline Constructive (M) & .36 & .58 & 2.38 \\
\hline Interactive (M) & .22 & .36 & 2.14 \\
\hline
\end{tabular}

Note. $\mathrm{N}$ is used to represent the sample size of higher education teachers in each cluster. $\mathrm{M}$ is used to represent the mean scores of the four types of initiated digital learning activities in each of the three clusters. Mean scores could range from 0-4.

\section{Discussion}

\subsection{Values, goals, and results of education}

The digital educational paradigm is building on the "new/old" dichotomy, where the "old" is not just outdated, but obsolete, not in demand in new historical circumstances, no longer valuable, and the "new" has no analogues in the past, and unequivocally innovative.

Nevertheless, traditional "paper" pedagogy is not homogeneous.

Remarks about formalism, reproductiveness, neglect of needs and personal interests, lagging behind in solving vital problems of a rapidly changing life are fair in relation to the formative approach, as a rule, associated with a knowledge-oriented paradigm, and, vice versa, are successfully eliminated in developmental teaching in a personality-oriented paradigm, independently of the presence/absence of digital technologies in the learning structure.

Creativity, critical thinking, communication, etc., considered the competencies of the twenty-first century, in fact, correlate with the personality functions of criticism, creativity (or creatively transforming function), focus on the neighbor, identified by the authors of personality-oriented education, as an inherent worth and a source of their development, etc. [12].

When describing the digital education model, substantive and technological issues are revealed in detail ("What to teach?" and "How to teach?"), opposed to the issue "For what to teach?". What is the ultimate goal of digital education? What image of a person is the new digital paradigm oriented towards? On the basis of what values and in what semantic space are the content deployed and technologies applied? Abilities to adapt to change, work in a team, be sociable and effective, even if they are developed to the maximum, do not automatically turn an individual into a free, spiritual, moral person, who can "develop an idea about himself" and "redefine his destiny" (V. C. Bibler). Without a clear definition of the place of eternal universal human values among the value orientations of education, it is impossible to fully guarantee that there will not be a return towards the "thing", in terms of M. M. Bakhtin, understanding of man, "falling out" of culture.

The personality-oriented paradigm declares the importance of developing such personality functions as selectivity, meaning-creation, ensuring the level of spirituality of life [12], and the similar things, referring to the nuclear values of Good, Truth, and Beauty.

We should notice that in the knowledge paradigm, humanistic ideals retain their role as the highest regulators. 


\subsection{The content of the educational process}

Genuine education not only satisfies needs but also shapes them. Therefore, one of the most important didactic questions - "WHAT will be presented to the student?" - always at the forefront of professional discussion. The large variability of digital content makes this issue more relevant than ever. Will the content selection criteria be blurred? What fate will be in the invariant component of educational content in the space of digital platforms that are not affiliated with educational institutions?

The individual approach does not imply transferring the educational process to a personally-semantical level. It doesn't create the appropriate situations during which the student would be able to gain not only the learning and actioning experience but also the emotional experience and emotional-valuable relations (I. Ya. Lerner, M. N. Skatkin). It should be pointed out that it is the last type of experience that helps to form the human's personal experience, which, according to K. Rogers, is the only criteria of assessing life events.

Personality experience is associated with acts of personality choice. It is known that personal choice is associated with alternativeness and responsibility, subjective activity, and is based on values.

The didactic nature of the content in the personality-oriented educational process allows you to make a personal choice, moreover, teaches you to make it. Culture is

symbolic and cultural texts should be understood individually. This is fundamentally a spiritual act that requires a spiritual search. It presupposes subject activity and personifies responsibility for the choice being made. The dialogue organization of the content reflects the alternativeness of the semantic space of culture, including the student in a large cultural dialogue. The cultural space is full of creative values. The highest humanistic values that are found in all peoples and in all epochs are transformed into eternal universal ones, as stable dominants of human culture. The loss of the cultural basis turns into a loss of ability for high identification.

However, values are not in a hierarchical relationship with each other in the cultural space. They are arranged in a hierarchy in the personality of a person who participates in a culture. In the personality-oriented educational process, the values of students always get challenged by new semantics included in the dialogue system and appearing during conversations. The personality of the teacher plays a great role here. Firstly, the teacher constructs educational content and dialogue activities in the educational process in such a way that the value aspect of comprehending the content is actualized and the nuclear values of a culture are manifested. Secondly, the teacher himself is the bearer of values, and his personal values become one of the tuning forks by which the students' personal value systems are "tuned in". The teacher's personal values must be formed and stable by the time of the pedagogical debut, and the eternal universal values in it must take the place of a system-forming element. Thus, in the personality-oriented paradigm, it is impossible to reduce the role of a teacher to support, to tutoring, which is assigned to him in digital education.

Also, it should be noted that the principle of organizing the thinking of representatives of the digital generation is more networked than hierarchical. Generic/species characters are of equal order [13]. This creates great risks of relativism, including moral relativism.

During digital transformation, they are multiplied by risks related to educational accessibility and technological inequality. This is a complex problem [14, 15], as well as the problem of learning from digital media [16]. It has been proven that reading linear texts from electronic media leads to worse reading comprehension [17].

In this respect, the digital substance of the content loses not only to the content in the personality-oriented paradigm but also in the knowledge-oriented paradigm. The use of digitized text in the context of a student-oriented educational process complicates 
comprehension and reflection since it becomes difficult to build a mental map of the text: only one page is physically available to the reader at any time of reading. Tasks for which it is necessary to integrate information from spatially distant places in the text (when associations are established, comparisons are made, meanings are extracted, etc.), require additional cognitive costs in terms of mental reconstruction of the text.

\section{Conclusions}

The transformation of education taking place in the context of digitalization affects the fundamental foundations of the existence of educational paradigms. The correlation of the goals and results of education with certain values, the principles and methods of organizing the content, established in the pedagogical context, should determine the appropriateness and measure of the use of digital technologies, and not vice versa.

At this stage of digitalization, there is a lack of clarity in determining the place of eternal universal values in the digital approach to education. There is a doubt about the relevance of the image of a spiritual and moral person who interiorizes the highest humanistic values embodied in a culture and makes free personal choices in the cultural space, while this image of a person determines the direction of pedagogical efforts in a personality-oriented paradigm. Personality-oriented education is faced with the task of preserving the invariant core of educational content and scientific and didactic criteria for the selection of content in the context of the large variability of digital content, ensuring the place of the personal experience of students and teachers in the structure of content and access to cultural texts. The problem of educational work with voluminous linear texts (which, as a rule, are cultural texts) on electronic media, associated with the difficulty of understanding, comprehending, and reflecting when reading from a screen, requires a solution. The tendency towards individualization of the orientation of education, and not to the strengthening of the personality orientation, traises concern. This can lead to a "wide choice in a narrow window of opportunity" effect.

\section{References}

1. N.C. Burbules, G. Fan, Ph. Repp, Geography and Sustainability 1(2), 93-97 (2020). doi: 10.1016/j.geosus.2020.05.001

2. I. Engeness, M. Nohr, Cultural-Historical Psychology 3(16), 71-82 (2020). doi: 10.17759/chp.2020160308

3. D. Golan, M. Barzillai, T. Katzir, Computers \& Education 126, 346-358 (2018). doi: 10.1016/j.compedu.2018.08.001

4. A.A. Grekova, Vestnik of the South Ural State University. Series: Psychology 1(12), 28-38 (2019)

5. V. Kuznetsova, I. Azhmukhamedov, Advantages and risks of using the digital educational environment, In: VI International Forum on Teacher Education, Kazan Federal University, 1369-1381 (2020). doi: 10.3897/ap.2.e1369

6. F.V. Lim, W. Toh, Journal of Information Literacy 2(14), 24-43 (2020). doi: $10.11645 / 14.2 .2701$

7. A. Lohr, M. Stadler, F. Schultz-Pernice, O. Chernikova, M. Sailer, F. Fischer, M. Sailer, Computers in Human Behavior 119, (2021). doi: 10.1016/j.chb.2021.106715

8. A. Mangen, B.R. Walgermo, B. Kolbjørn, International Journal of Educational Research 58, 61-68 (2013). doi: 10.1016/j.ijer.2012.12.002 
9. E.M. Molchan, Vestnik of Moscow Region State University. Series: Philosophy 2, 5566 (2019) doi: 10.18384/2310-7227-2019-2-55-66

10. I.Ya. Murzina, Education and Science 22(10), 90-115 (2020). doi: 10.17853/19945639-2020-10-90-115

11. T N. Noskova, T.B. Pavlova, Vestnik of State Pedagogical University 1(206), 94-103 (2020) doi: 10.23951/1609-624X-2020-1-94-103

12. A.V. Ostrovsky, M.V. Kudina, Public administration. E-journal 78, 229-244 (2020)

13. D. Radovanović, B. Hogan, D. Lalić, New Media \& Society 17(10), 1733-1749 (2015). doi: 10.1177/1461444815588323

14. V.V. Serikov, Vestnik of the Volgograd State Pedagogical University 2(106), 30-35 (2016).

15. B. Tripathy, S. Raha, International Research Journal of Engineering and Management Studies 5(3), 1-4 (2019).

16. A.Yu. Uvarov, E. Gejbl, I.V. Dvoreckaya, I.M. Zaslavsky, I.A. Karlov, T.A. Mertsalova, P.A. Sergomanov, I.D. Frumin, Difficulties and prospects of the digital transformation of education (Publishing House of Higher School of Economics, Moscow, 2019)

17. Sh. Young, Educational Studies Moscow 4, 21-43 (2018). doi: 10.17323/1814-95452018-4-21-43 